\title{
Reference percentiles of FEV1 for the Canadian cystic fibrosis population: comparisons across time and countries
}

\author{
Sang-Ook Kim, ${ }^{1,2}$ Mary Corey, ${ }^{2}$ Anne L Stephenson, ${ }^{3,4}$ Lisa J Strug ${ }^{1,2}$
}

\begin{abstract}
- Additional material is published online only. To view please visit the journal online (http://dx.doi.org/10.1136/ thoraxjnl-2017-210899)
\end{abstract}

${ }^{1}$ Division of Biostatistics, Dalla Lana School of Public Health, University of Toronto, Toronto, Canada

${ }^{2}$ Research Institute, The Hospital for Sick Children, Toronto,

Canada

${ }^{3}$ Department of Respirology, Adult Cystic Fibrosis Program, St. Michael's Hospital, Toronto, Canada

${ }^{4}$ Institute of Health Policy, Management and Evaluation, University of Toronto, Toronto, Canada

\section{Correspondence to} Dr Anne L Stephenson, St. Michael's Hospital, Toronto, ON M5B 1W8, Canada; stephensona@smh.ca and Dr Lisa J Strug, Research Institute, Hospital for Sick Children, Toronto, ON M5G 1X8, Canada; lisa.strug@utoronto.ca

Received 11 August 2017 Revised 1 December 2017 Accepted 5 January 2018 Published Online First 6 February 2018

Check for updates

To cite: Kim S-O, Corey M, Stephenson AL, et al. Thorax 2018:73:446-450.

\section{ABSTRACT}

Background Forced expiratory volume in $1 \mathrm{~s}$ (FEV1) indicates lung health in cystic fibrosis (CF). FEV1 is commonly communicated as a per cent predicted of a healthy individual sharing the same age, sex, race and height. CF-specific reference equations are complementary and calibrate a patient's FEV1 to that of their CF peers.

Objectives (1) To derive Canadian CF-specific FEV1 reference percentiles (FEV1\%iles), (2) characterize how they have changed over time and (3) compare the Canadian FEV1\%iles to those for USA and European CF populations.

Method CF FEV1\%iles are calculated using the Canadian CF Registry and quantile regression.

Results The Canadian FEV1\%iles demonstrated better lung function in more recent time periods within Canada, especially below the $50 \%$ percentile and in males. When compared to USA and European FEV1\%iles for the same time period, Canadian FEV1\%iles were higher.

Conclusion CF-specific FEV1\%iles can provide useful information about changes in lung health. An online calculator (available at cfpercentile.research.sickkids.ca) makes these FEV1\%iles accessible.

\section{INTRODUCTION}

Forced expiratory volume in $1 \mathrm{~s}$ (FEV1) is the single most important predictor of survival in cystic fibrosis $(\mathrm{CF}) .^{1-3}$ As a result, it is routinely monitored or used as an outcome measure. ${ }^{4-7}$ Typically, FEV1 is reported as a percentage (FEV1pp) compared with a healthy population based on age, sex, height and race. ${ }^{8-13}$ Comparing with a healthy population reflects the CF goal of achieving normal lung function throughout life. Although this comparison is a useful reflection of morbidity, comparing the FEV1 of a patient with CF to others with CF can provide additional information. ${ }^{14-17}$ For example, a 50-year-old patient with CF with an FEV1 that is $50 \%$ of normal may be considered to have moderately severe lung disease, although this individual has lung function that is higher than most of the CF population at that age.

CF-specific FEV1 percentiles (FEV1\%iles) have been previously derived for the USA, French and European CF populations ${ }^{1718}$ and have been used as the basis for lung disease phenotypes in gene modifier studies. Because of international differences in improvements in lung function and survival, it is challenging to apply FEV1\%iles from one country

\section{Key messages}

What is the key question?

- How does the lung health of the Canadian cystic fibrosis (CF) population compare to the USA and European populations, and how has it changed over time within Canada?

What is the bottom line?

- The level of lung function of the Canadian CF population was generally higher over a time period when survival was also steadily improving and generally higher than the levels in the USA and Europe over comparable periods.

Why read on?

- This study provides CF-specific forced expiratory volume in $1 \mathrm{~s}$ reference equations along with an online calculator to monitor the lung function of patients with $\mathrm{CF}$, which can be interpreted outside of the context of a healthy population.

derived in perhaps earlier decades to countries that have different survival patterns.

The objectives of this study were to (1) derive contemporary Canadian CF-specific reference FEV1\%iles, (2) assess changes in the Canadian CF FEV1 distribution over time and (3) compare Canadian CF percentiles with previously published USA and European CF percentiles.

\section{METHODS}

We used Canadian CF Registry (CCFR) data between 2008 and 2014 to estimate the contemporary Canadian CF-specific FEV1\%iles (see online supplementary file 1 and figure S1 in the online supplementary file 2). Each patient underwent informed consent.

For temporal comparison, CCFR data between 2000 and 2007 were used. For the comparison with the USA and Europe, we used CCFR with matching data collection periods (see online supplementary file 1).

To estimate CF-specific FEV1\%iles, we used quantile regression ${ }^{19}$ adjusting for age and/or height with smoothing, separately by sex. Cluster bootstrap ${ }^{20}$ was used for variance estimation and we tested for the difference in the area under the curve for the temporal and intercountry 
comparisons with Bonferroni correction for multiple hypothesis testing. We compared our quantile regression approach with Generalised Additive Models for Location, Scale and Shape (GAMLSS; see online supplementary file 1$)^{21}$

\section{RESULTS}

\section{Contemporary Canadian cohort: 2008-2014}

The contemporary Canadian cohort included 3784 (45.8\% female) patients contributing 20169 annual clinical measurements taken between 2008 and 2014 (see figure S1 in the online supplementary file 2). Baseline demographic and clinical characteristics are shown in table S1 in the online supplementary file 3.
CF-specific FEV1\%iles after adjusting for age are shown in figure $1 \mathrm{~A}$ (solid line). Lung function increases continuously throughout childhood and adolescence and declines in adulthood. The median FEV1 peak in males was $3.31 \mathrm{~L}$ reached at 18 years of age; for females, it was $2.52 \mathrm{~L}$ at 15 years. The heightadjusted CF-specific FEV1\%iles are displayed in figure 1B (solid line). Overall, FEV1 increased almost linearly with height at each percentile.

The Canadian CF-specific reference equations were computed by adjusting for age and sex. We show FEV1\%iles by height for Canadian patients with CF aged 12, 18 or 35 years, separately by sex in figure S2 in the online supplementary file
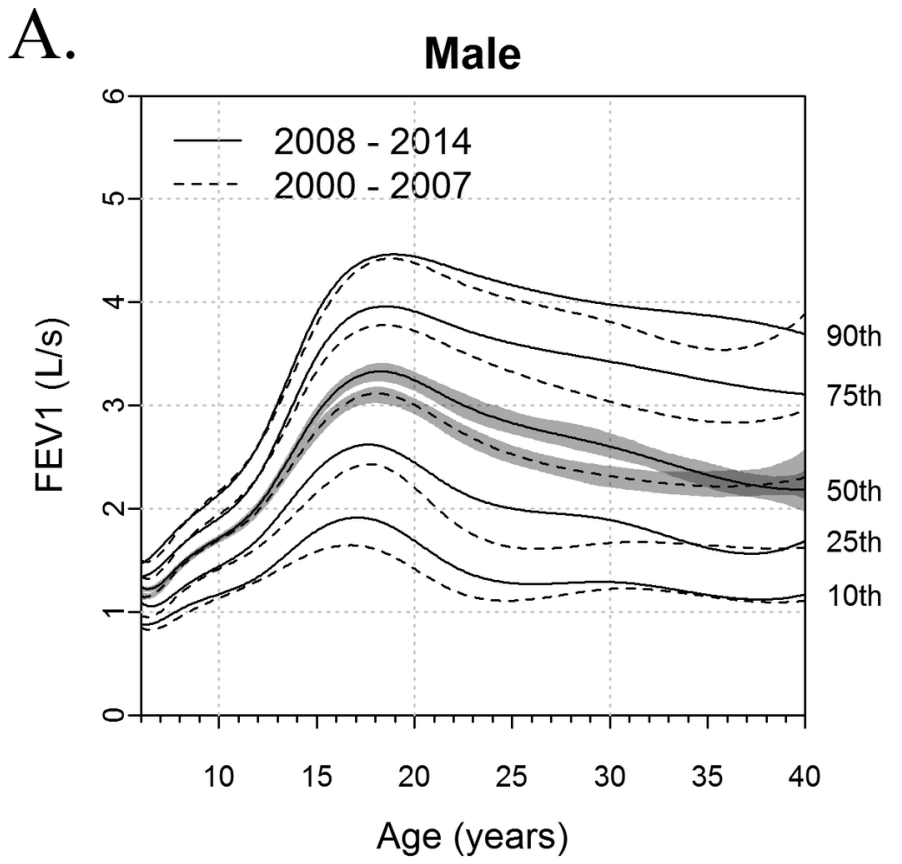

B.

Male

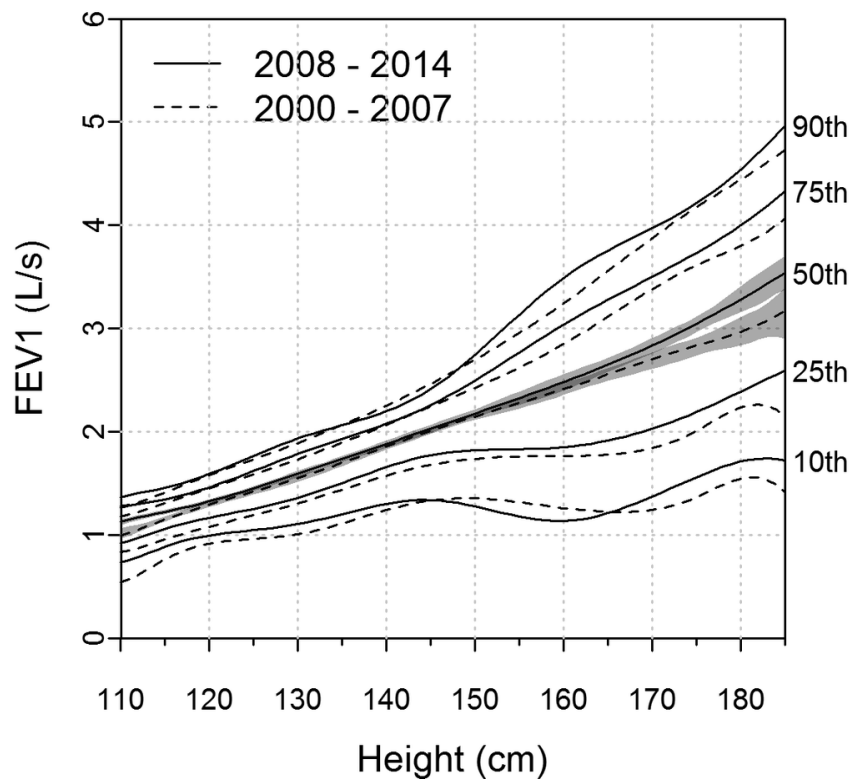

Female

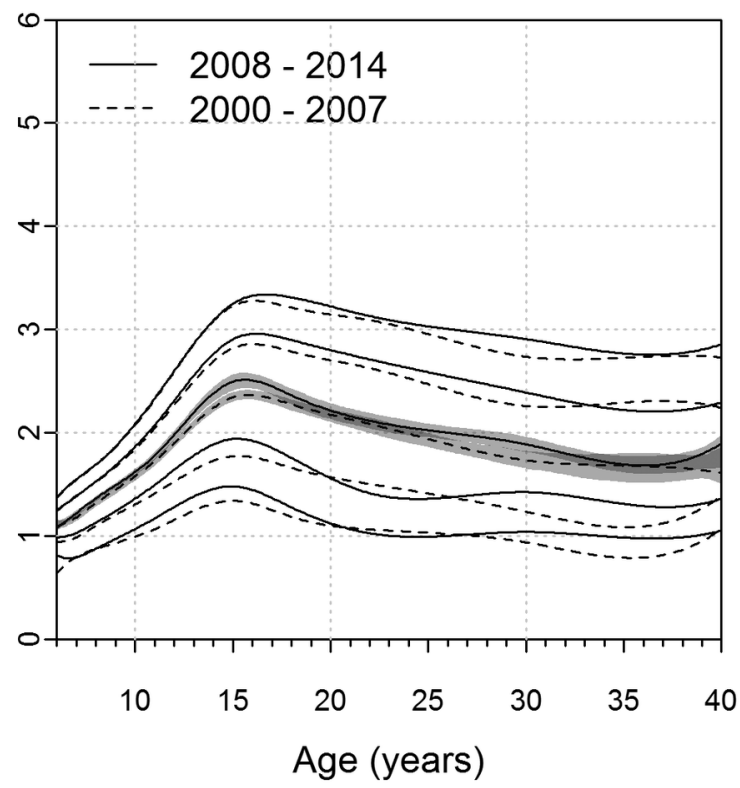

Female

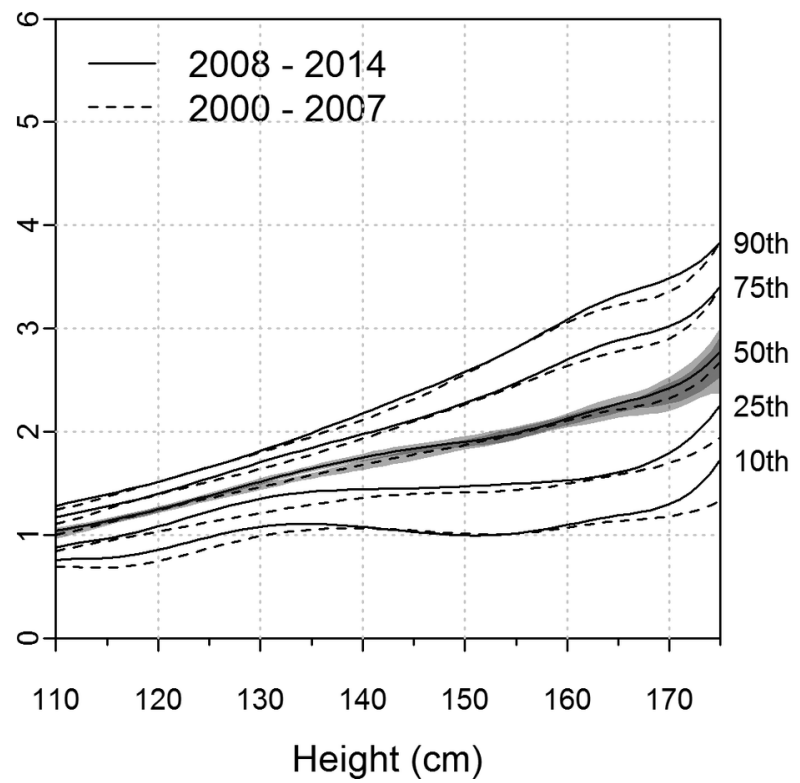

Figure 1 Comparison of Canadian cystic fibrosis-specific forced expiratory volume in $1 \mathrm{~s}$ (FEV1) percentiles between two time periods, 2000-2007 (dashed line) and 2008-2014 (solid line) adjusted for (A) age or (B) height, stratified by sex; 10th, 25th, 50th, 75th and 90th percentiles are shown. The grey area displays the $95 \%$ pointwise confidence band for the median FEV1 for each time period. 
A.

Male

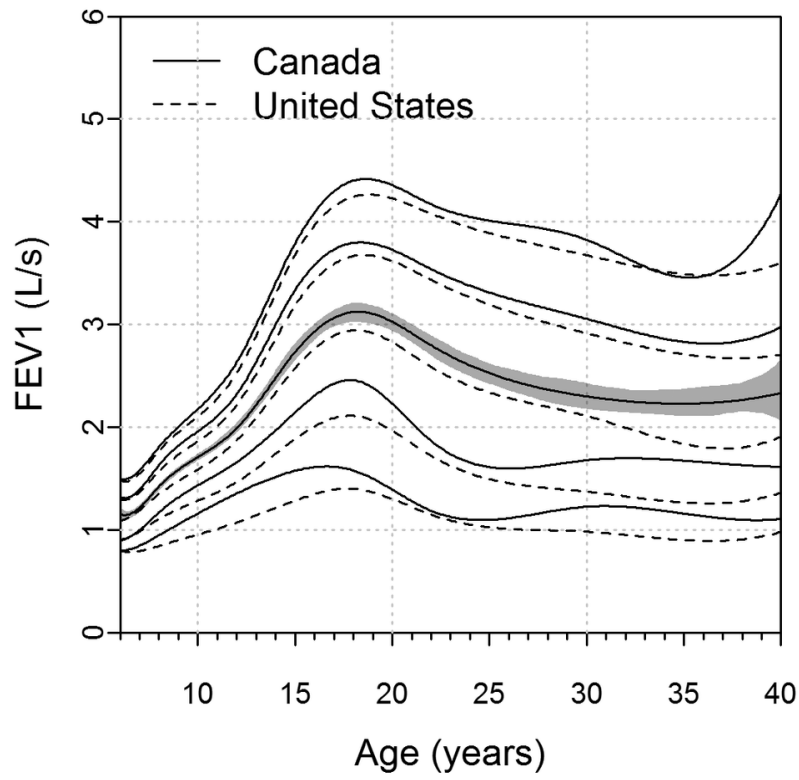

B.

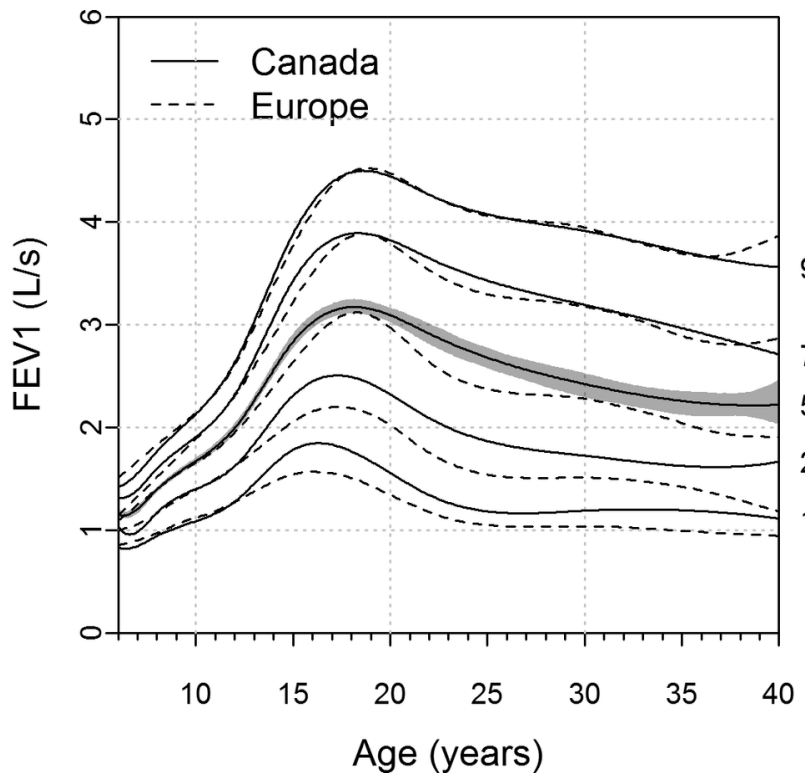

Female

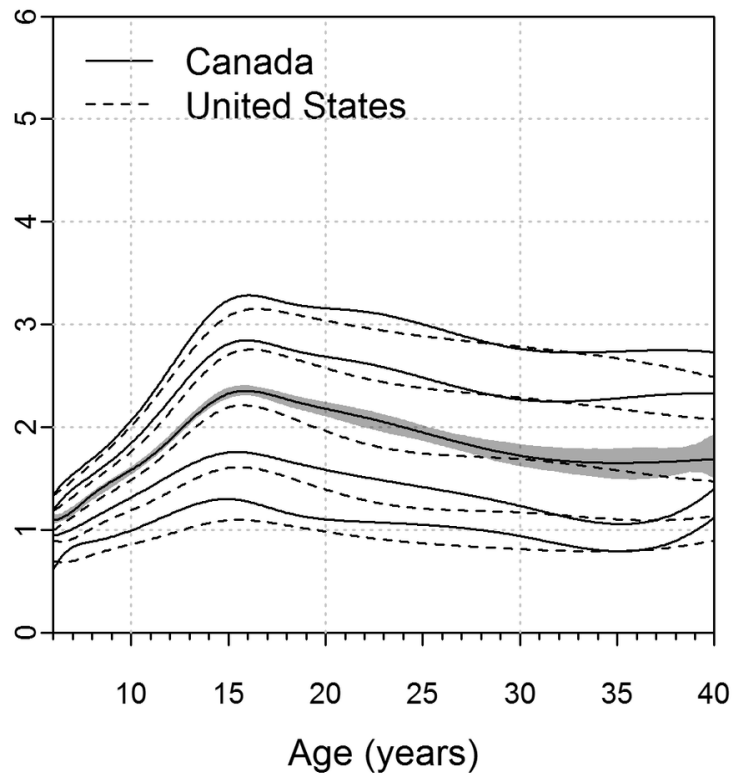

Female

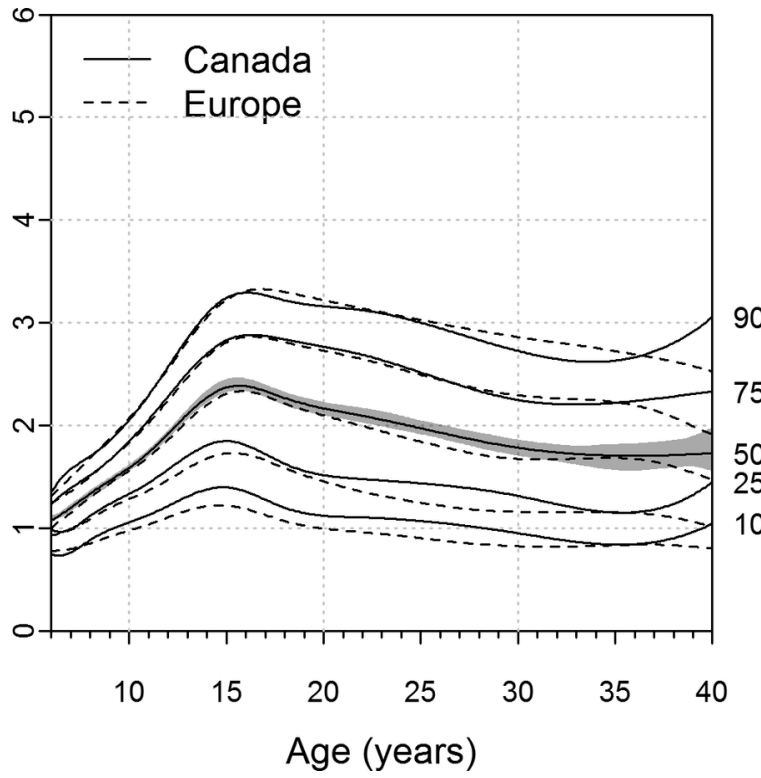

Figure 2 Comparison of age-adjusted cystic fibrosis-specific forced expiratory volume in $1 \mathrm{~s}$ (FEV1) percentiles between (A) Canada (solid line) and USA (dashed line) (2001-2006) and (B) between Canada (solid line) and European countries (dashed line) (2004-2010), stratified by sex; 10th, 25th, 50th, 75th and 90th percentiles are shown. The grey area displays the 95\% pointwise confidence band for the Canadian median FEV1. The graph coordinates for the European FEV1 percentiles were approximated using Plot Digitizer (V.2.6.8) and then smoothed.

4. With the reference equations, one can estimate the percentile of FEV1 measurement of a patient with CF given their age and height. For example, an 18-year-old male patient who has a standing height of $172 \mathrm{~cm}$ and FEV1 of $3.29 \mathrm{~L}$ at the clinical visit has an estimated median FEV1. In other words, $50 \%$ of the 18 -year-old male patients with CF with a height of $172 \mathrm{~cm}$ had lower FEV1 than the hypothetical patient.

We used GAMLSS to estimate CF-specific FEV1\%iles adjusted for age or height (see figure S5 in the online supplementary file 5). Quantile regression and GAMLSS yielded similar results; minor differences existed mainly below the first and above the 99th percentile.

\section{Changes over time within Canada}

Figure 1A shows the age-adjusted Canadian CF-specific FEV1\%iles obtained from two time periods: 2000-2007 and 2008-2014. For males, the level of lung function at each CF-specific FEV1\%ile was significantly higher in the more recent time period compared with the earlier time period $(\mathrm{P}<0.01)$, reflecting an overall improvement in lung function at the population level. Ninety-nine per cent confidence bands (see figure S4 
in the online supplementary file 6) showed significant differences at various age ranges by percentile and sex (see online supplementary file 1). For the female CF population, improvements were significant after multiple hypothesis testing correction at the 10th, 25th and 50th percentiles $(\mathrm{P}<0.01)$ but not at the 75th and 90 th percentiles $(\mathrm{P}=0.015$ and 0.024 , respectively).

Since spirometry is influenced by height, ${ }^{17}$ we used the height-adjusted Canadian CF-specific FEV1\%iles to compare two time periods, 2000-2007 and 2008-2014 (figure 1B). For the male Canadian CF population, the improvement in lung function over time was significant at each height percentile $(\mathrm{P}<0.01)$, whereas, for females, there was no statistically significant difference between the two time periods for any percentile.

\section{International comparisons}

Cumulative age-adjusted CF-specific FEV1\%iles for the CF population in Canada were significantly higher than those in USA for both sexes for the 2001-2006 time period $(\mathrm{P}<0.01$; figure 2A). Ninety-nine per cent confidence bands (see figure S6 in the online supplementary file 7) showed significant differences at various age ranges (see online supplementary file 1). For example, an 18-year-old Canadian male patient with CF with $2.37 \mathrm{~L}$ of FEV1 was at the 25th CF-specific percentile when Canadian reference equations were applied, whereas USA reference equations overestimated his FEV1\%ile at the 32 nd percentile. Differences appeared more pronounced at the lower percentiles (10th and 25th percentiles) than higher percentiles (75th and 90th percentiles) for males. Cumulative height-adjusted CF-specific FEV1\%iles were significantly higher in the Canadian CF population than the USA for both sexes ( $\mathrm{P}$ values $<0.01$; see figure $\mathrm{S} 5$ in the online supplementary file 8$)$.

Canadian cumulative age-adjusted FEV1\%iles were significantly higher than those for Europe ${ }^{18}$ for both sexes $(\mathrm{P}<0.01$; figure 2B) except for the 90th percentile. Ninety-nine per cent confidence bands (see figure S7 in the online supplementary file 9) showed significant differences at various age ranges (see online supplementary file 1). The 25th percentile of FEV1 for Canadian males attained its peak of $2.51 \mathrm{~L} / \mathrm{s}$ at the age of 17 years, whereas for European males, the peak was $2.21 \mathrm{~L} / \mathrm{s}$, reached at the same age. For females, the 25 th percentile peaks for FEV1 were $1.85 \mathrm{~L} / \mathrm{s}$ and $1.72 \mathrm{~L} / \mathrm{s}$, respectively, for Canadians and Europeans, both at the age of 15 years.

\section{DISCUSSION}

We developed contemporary Canadian CF-specific reference equations for lung function, which can be used to understand the severity of lung disease for a contemporary group of individuals living with CF today compared with their peers. These data demonstrate significant improvements in lung function within the Canadian CF population over time, likely due to early diagnosis, multidisciplinary care, better nutritional status early in life, Pseudomonas aeruginosa eradication strategies and aggressive treatment of pulmonary exacerbations. ${ }^{22}$

Improvements in lung function across all selected percentiles were noted for males when comparing data from 2000 to 2007 versus 2008-2014, while statistically significant results were seen only at lower percentiles for females. It is possible that aggressive nutritional supplementation, particularly early in life, may result in more substantial increases in growth and lung development in males compared with females. Given that more severely affected patients lived longer, ${ }^{23}$ it is impressive that the population level of lung function was as good or better.
Canadian patients generally had significantly higher FEV1 across the spectrum of percentiles and ages compared with the USA and European populations. Furthermore, the data showed a wider gap in those with lower lung function compared with those at higher percentiles. Those with lower lung function are at higher risk of death, and therefore, the fact that Canadians have higher level of lung function in the lower percentiles is consistent with better overall survival in Canada compared with the USA. ${ }^{24}$ The high fat diet was implemented earlier in Canada compared with the USA which may explain the higher level of lung function in Canadians with $\mathrm{CF}^{24}$ These cross-population differences highlight the necessity for CF-specific reference equations that are population specific. Moreover, the differences between populations vary at different ages and heights. Therefore, the impact of estimating age and height-adjusted FEV\%ile for Canadian patients with CF from USA reference equations would differ at different ages and heights.

There are some study limitations to note. First, our analysis did not directly embed the correlation structure in the estimation of FEV1\%iles. However, balancing the measurements by using one measurement/patient/year attenuates any resulting bias, ${ }^{25}$ and variance estimation using a cluster bootstrap ensures statistical testing validity.

Second, selecting the first stable FEV1 measurement/patient/year rather than all measurements impacts the correlation strength ${ }^{26}$ but reduces estimation bias of the FEV1\%iles since sicker patients visit the clinics more frequently. Third, our final model used quantile regression rather than GAMLSS since our interest was on percentile estimation, ${ }^{27}$ but the choice of regression methodology was inconsequential to our conclusions. Lastly, as in other studies, ${ }^{17}{ }^{18}$ the interior knots for smoothing of the percentile curves were chosen based on visualisation, influencing the model fit. A sensitivity analysis that varied the number and location of knots and implementation of an approach that chooses roughness penalties through cross-validation $^{28}$ suggested these choices did not impact the study conclusions.

The Canadian CF-specific FEV1\%iles provide a complementary approach to using the healthy population as a standard for $\mathrm{CF}$ lung health. These Canadian CF-specific FEV1\%iles are available as an interactive tool (cfpercentile.research.sickkids.ca) to assess a patient's relative state of lung disease against Canadian peers and will facilitate international comparisons. Moreover, these Canadian CF-specific FEV1 percentiles now provide a contemporary reference to calculate updated and improved lung disease phenotypes for genetic studies, the goal of future work.

Acknowledgements The authors would like to thank Cystic Fibrosis Canada who maintains the Canadian Cystic Fibrosis Registry, in particular Dr Denise Mak and Jenna Sykes for their assistance with the dataset, clinics, patients and their families for their ongoing participation in the Registry.

Contributors All authors contributed to the data analysis, manuscript preparation and revisions. All authors have approved the final version of this paper.

Funding This work was funded by grants to LSS from the Canadian Institutes of Health Research (CIHR; MOP-258916), the Natural Sciences and Engineering Research Council of Canada (NSERC; 371399-2009) and Cystic Fibrosis Canada (\#2626).

Competing interests None declared.

Ethics approval Research Ethics Board of Hospital for Sick Children, Toronto, Canada.

Provenance and peer review Not commissioned; externally peer reviewed.

(C) Article author(s) (or their employer(s) unless otherwise stated in the text of the article) 2018. All rights reserved. No commercial use is permitted unless otherwise expressly granted. 


\section{REFERENCES}

1 Schluchter MD, Konstan MW, Davis PB. Jointly modelling the relationship between survival and pulmonary function in cystic fibrosis patients. Stat Med 2002;21:1271-87.

2 Kerem E, Reisman J, Corey M, et al. Prediction of mortality in patients with cystic fibrosis. N Engl J Med 1992;326:1187-91.

3 Corey $\mathrm{M}$, Edwards L, Levison $\mathrm{H}$, et al. Longitudinal analysis of pulmonary function decline in patients with cystic fibrosis. J Pediatr 1997;131:809-14.

4 Ramsey BW, Astley SJ, Aitken ML, et al. Efficacy and safety of short-term administration of aerosolized recombinant human deoxyribonuclease in patients with cystic fibrosis. Am Rev Respir Dis 1993;148:145-51.

5 Konstan MW, Byard PJ, Hoppel CL, et al. Effect of high-dose ibuprofen in patients with cystic fibrosis. N Engl J Med 1995;332:848-54.

6 Ramsey BW, Pepe MS, Smith AL, et al. Efficacy and safety of chronic intermittent administration of inhaled tobramycin in patients with cystic fibrosis. N Engl J Med 1999;340:23-30.

7 Saiman L, Marshall BC, Mayer-Hamblett N, et al. Azithromycin in patients with cystic fibrosis chronically infected with Pseudomonas aeruginosa: a randomized controlled trial. JAMA 2003;290:1749-56.

8 Hankinson JL, Odencrantz JR, Fedan KB. Spirometric reference values from a sample of the general U.S. population. Am J Respir Crit Care Med 1999;159:179-87.

9 Knudson RJ, Slatin RC, Lebowitz MD, et al. The maximal expiratory flow-volume curve. Normal standards, variability, and effects of age. Am Rev Respir Dis 1976;113:587-600.

10 Stanojevic S, Wade A, Stocks J, et al. Reference ranges for spirometry across all ages: a new approach. Am J Respir Crit Care Med 2008;177:253-60.

11 Wang SS, O'Leary LA, Fitzsimmons SC, et al. The impact of early cystic fibrosis diagnosis on pulmonary function in children. J Pediatr 2002;141:804-10.

12 Wang $X$, Dockery DW, Wypij D, et al. Pulmonary function between 6 and 18 years of age. Pediatr Pulmonol 1993;15:75-88.

13 Quanjer PH, Stanojevic S, Cole TJ, et al. Multi-ethnic reference values for spirometry for the 3-95-yr age range: the global lung function 2012 equations. Eur Respir J 2012:40:1324-43
14 Lebowitz MD, Sherrill DL. The assessment and interpretation of spirometry during the transition from childhood to adulthood. Pediatr Pulmonol 1995:19:143-9.

15 Borsboom GJ, Van Pelt W, Quanjer PH. Pubertal growth curves of ventilatory function: relationship with childhood respiratory symptoms. Am Rev Respir Dis 1993; 147:372-8

16 Merkus PJ, Tiddens HA, de Jongste JC. Annual lung function changes in young patients with chronic lung disease. Eur Respir J 2002;19:886-91.

17 Kulich M, Rosenfeld M, Campbell J, et al. Disease-specific reference equations for lung function in patients with cystic fibrosis. Am J Respir Crit Care Med 2005;172:885-91.

18 Boëlle PY, Viviani L, Busson PF, et al. Reference percentiles for FEV(1) and BMI in European children and adults with cystic fibrosis. Orphanet J Rare Dis 2012;7:64.

19 Koenker R, Bassett G. Regression quantiles. Econometrica 1978;46:33-50.

20 Sherman M, Cessie Sle, leCessie S. A comparison between bootstrap methods and generalized estimating equations for correlated outcomes in generalized linear models. Commun Stat Simul Comput 1997;26:901-25.

21 Rigby RA, Stasinopoulos DM. Generalized additive models for location, scale and shape (with discussion). Journal of the Royal Statistical Society: Series C 2005;54:507-54

22 Cohen-Cymberknoh M, Shoseyov D, Kerem E. Managing cystic fibrosis: strategies that increase life expectancy and improve quality of life. Am J Respir Crit Care Med 2011;183:1463-71.

23 Stephenson AL, Tom M, Berthiaume Y, et al. A contemporary survival analysis of individuals with cystic fibrosis: a cohort study. Eur Respir J 2015;45:670-9.

24 Stephenson AL, Sykes J, Stanojevic S, et al. Survival comparison of patients with cystic fibrosis in canada and the united states: a population-based cohort study. Ann Intern Med 2017; 166:537-46.

25 Fitzmaurice G, Laird N, Ware J. Applied longitudinal analysis. New York: Wiley, 2004.

26 Taylor-Robinson D, Whitehead M, Diderichsen F, et al. Understanding the natural progression in \%FEV1 decline in patients with cystic fibrosis: a longitudinal study. Thorax 2012:67:860-6.

27 Kneib T. Beyond mean regression (with discussion and rejoinder). Stat Model 2013;13:275-385

28 Muggeo VMR, Sciandra M, Tomasello A, et al. Estimating growth charts via nonparametric quantile regression: a practical framework with application in ecology. Environ Ecol Stat 2013;20:519-31. 